\title{
OFDM: A Mathematical Review
}

\section{Praveen Kumar Malik ${ }^{1}$, M P Tripathi ${ }^{2}$}

${ }^{1}$ Deptt. of ECE, Inderprastha Engineering College, Ghaziabad UP India.

${ }^{2}$ Deptt. of ECE, NIT Patna, Patna, Bihar, India.

E-mail: pkmalikmeerut@gmail.com, munish@nitp.ac.in

Published Online: 28 December, 2017.

The Author(s) 2017. This article is published with open access at www.chitkara.edu.in/publications

\begin{abstract}
Mathematical review of the Orthogonal Frequency Division Multiplexing is demonstrated in terms of Inter symbol interference, Multi carrier modulated system and cyclic prefix. Modeling of the mathematical equation of the Orthogonal Frequency Division Multiplexing, Inverse fast Fourier transform and fast Fourier transform is explained with the suitable example using MATLAB. Bit error rate performance of OFDM is also presented with the help of statistical computation
\end{abstract}

Keywords: Cyclic prefix, Inter symbol interference, Multi carrier modulated systems.

\section{INTRODUCTION}

Orthogonal frequency division multiplexing (OFDM) is a broadband key technology which is employed in "4G wireless communication system". It is also used in "Long term evolution" (LTE) 4G cellular standard and Worldwide interoperability for microwave access (Wi max) also. These are now day's dominants standards and both are based on OFDM.

LTE -A long term evolution advanced which is latest communication standard in broad band is also based on OFDM. LTE is a wireless communication standard which can support a large bandwidth. In general GSM has a bandwidth of about $200 \mathrm{KHz}$ but OFDM can have bandwidth of about $100 \mathrm{MHz}$. Naturally data rate in OFDM will be higher which is used in $3 \mathrm{G}$ and $4 \mathrm{G}$ to enable data rate of up to $100 \mathrm{MBps}$ or more then $500 \mathrm{MBps}$. Several IEEE Wi-Fi wireless local area networks WLAN $(802.11,802.11 \mathrm{G}$, $802.11 \mathrm{~N}, 802.11 \mathrm{AC}$ ) standards are used for high data rate which are based on the OFDM.

\section{INTER SYMBOL INTERFERANCE}

In traditional communication systems let we have a bandwidth and there is a single carrier frequency for it. For better understanding of this concept, let Bandwidth of a channel is $1 \mathrm{MHz}$, therefore symbol time

Journal on Today's Ideas Tomorrow's Technologies, Vol. 5, No. 2, December 2017 pp. $97-111$

CHITKARA 司 
Malik, P. K.

Tripathi, M. P. $\quad T_{S}=1 / B=1 / 20 M h z=0.05 \mu s$. However in a typical communication system delay spread is about $2 \sim 3 \mu s\left(T_{d} \approx 2 \sim 3 \mu s\right)$ which says that symbol time is much less than delay spread $T_{S}<<T_{d}$. Hence, whenever there is symbol time is less then delay spread time then we have "inter symbol interference" (ISI). This leads to a problem in communication system which degrades the performance of the wireless communication system. As bandwidth increases our symbol time decreases. This is a significant problem in the broadband channel design.

\section{PRINCIPLE TO AVOID ISI}

To avoid the "Inter symbol interference" we split the large bandwidth into smaller bands or sub bands. Therefore in each sub band we will have individual subcarrier. Let $\mathrm{N}$ sub bands are there then bandwidth of each sub band will be . For example $\mathrm{B}=20 \mathrm{MHz}, \mathrm{N}=1000,(\mathrm{~N}=$ No of sub bands) then bandwidth of per sub band $20 \mathrm{MHz} / 1000=20 \mathrm{KHz}$. Then symbols time will be $1 / 20 \mathrm{KHz}=0.05 \mathrm{~ms}=50 \mu \mathrm{s}$. As now delay spread time is more than symbol time $T \gg T_{d}$. Therefore there is no Inter symbol interference which is done with the help of multiple sub bands and sub carriers. Such a system with a multi sub bands and sub carrier is known as a multi carrier modulated system (MCM system) [1]. This is the principle basis of the orthogonal frequency division multiplexing.

\section{MULTI CARRIER MODULATED TRANSRECEIVER}

Let us assume for instant at fundamental frequency $F_{0}=B / N$ which can be written as $\ldots \ldots-2 F_{0},-F_{0}, 0, F_{0}, 2 F_{0} \ldots \ldots$ total of $\mathrm{N}$ subcarrier. There-

fore $\mathrm{K}^{\text {th }}$ subcarrier will be defined as $k \cdot F_{0}=k \frac{B}{N} \cdot \mathrm{K}^{\text {th }}$ subcarrier is given as $e^{j 2 \pi k F_{0}}$. Let the $\mathrm{K}^{\text {th }}$ symbol is given by $X_{k}$ then the transmitted signal on $\mathrm{K}^{\text {th }}$ subcarrier will be $S_{k}^{t}=X_{k} e^{j 2 \pi k F_{0} t}$. Net transmitted multi carrier modulated signal will be sum of transmit signals across all $\mathrm{N}$ subcarrier and will be defined as: -

$\sum_{k} X_{k} e^{j 2 \pi k F_{0} t}--------------------(1)$

At the receive signal in equation 1 if we ignore the noise will be 
$y(t)=\sum_{k} X_{k} e^{j 2 \pi k F_{0} t} \quad$ (Similar to Fourier series expansion)

Now how to recover these symbols, i.e. to extract (also known as symbol on lth subcarrier) $X_{l}=F_{0} \int_{0}^{1 / F_{0}} e^{-j 2 \pi l F_{0} t} y(t) d t$ known as coefficient of the signal $y(t)$. Where is the fundamental time period of the signal. This will give

$X_{l}=F_{0} \int_{0}^{1 / F_{0}} e^{-j 2 \pi l F_{0} t} \sum_{k} X_{k} e^{j 2 \pi k F_{0} t} d t$

$X_{l}=\sum_{k} X_{k} F_{0} \int_{0}^{1 / F_{0}} e^{j 2 \pi(k-l) F_{0} t} d t--------------(2)$

As we know that $e^{j 2 \pi(k-l) F_{0} t}=\left\{\begin{array}{l}1, k=l \\ 0, k \neq l\end{array}\right\}$

Equation 2 will be:

$X_{l}=\sum_{k} X_{k} \delta(k-l)$

Therefore only the term $k=l$ will survive. This can be done for all the subcarrier to recover all the $\mathrm{N}$ symbols transmitted on the various subcarriers and termed as coherent demodulated system. At receiver we may employ coherent demodulation with $e^{-j 2 \pi l F_{0} t}$ to extract $X_{l}$. By correlating with $e^{-j 2 \pi l F_{0} t}$ or matched filtering with $e^{-j 2 \pi l F_{0}(T-t)}$ we can extract symbols on th subcarrier. This is the main principle of the multi carrier modulated system [2].

\section{OFDM TRANSMITTER AND RECEIVER MODEL}

\section{A. Transmitter schematic}

At transmitter we have $X(0), X(1), \ldots \ldots . . X(N-1)$ Symbols. Serial to parallel convertor also known as demultiplexing (DEMUX) is used at transmitter to load to the symbols onto the subcarrier. Then take N pt IFFT of these symbols $X(0), X(1), \ldots \ldots . . X(N-1)$ to generate transmit samples. $x(0), x(1), \ldots \ldots . . x(N-1)$ are known as transmit samples. These are now again converted back to parallel to serial stream/convertor to generate the 
Malik, P. K. Tripathi, M. P. serial stream of $x(0), x(1), \ldots \ldots . x(N-1)$. Now add the cyclic prefix (CP) $x(N-\bar{L}) \ldots \ldots \ldots \ldots . . . . x(N-1) x(0) x(1) \ldots \ldots . . x(N-1) \quad x(N-\bar{L}) \ldots \ldots \ldots \ldots . . . x(N-1) \quad$ are known as cyclic prefix. $\quad x(N-\bar{L}) \ldots \ldots \ldots \ldots . \ldots x(N-1) x(0) x(1) \ldots \ldots . . . x(N-1)$ these are the samples transmitted over the channel.

\section{B. Receiver Schematic}

At receiver we have samples over the wireless channel. $x(N-\bar{L}) \ldots \ldots \ldots \ldots \ldots(N-1) x(0) x(1) \ldots \ldots \ldots x(N-1)$ Therefore, first of all we will remove the cyclic prefix, because it consists of inter block interference. Now we have $y(0), y(1), \ldots \ldots . . . y(N-1)$ but these are in serial form. Then we can carry out a serial to parallel operation (de multiplexing). Perform the $\mathrm{N}$ pt FFT operation. Input to the FFT is received samples $y(0), y(1), \ldots \ldots . . . y(N-1)$ in parallel form. Output of the FFT will be $Y(0), Y(1), \ldots \ldots . . . Y(N-1)$ these are the FFT of received samples $y(0), y(1), \ldots \ldots . . . y(N-1)$. From these samples we have to detect symbols. Therefore, output of detector module will be $X_{h}(0), X_{h}(1), \ldots \ldots . . X_{h}(N-1)$ are known as detected symbols. It is not necessarily $X_{h}(k) \neq X(k)$ because there can be error. Then finally parallel to serial conversion (Multiplexing) is there to convert these back to serial symbol stream [3].

\section{ORTHOGONAL FREQUENCY DIVISION MULTIPLEXING}

OFDM is very efficient technique because it employs IFFT/FFT, fast efficient algorithm and no matrix inversion calculation is there. Orthogonal frequency division multiplexing is used in the following technologies: -

1. Modern wireless technology

2. 4 G Standards

3. LTE, Wi max, Wi Fi and 802.11 etc

ISI channel is described as

$y(k)=h(0) x(k)+h(1) x(k-1)+v(k)$

$y(k)=$ Received symbols at time $\mathrm{k}, x(k)=$ transmitted symbols at time $\mathrm{k}$, $x(k-1)=$ transmitted symbols at time $(k-1), v(k)$ is noise. OFDM overcomes ISI with very low complexity and it is very efficient scheme.

Let the following symbols are to be transmitted: $X(0), X(1), X(2), X(3)$

$\mathrm{N}=4$ Symbols and similarly 4 Subcarriers.

$X(l)$ is loaded on to the lth subcarrier. 
$\mathrm{N}$ point IFFT is taken at the transmitter

$x(k)=\frac{1}{N} \sum_{l=0}^{N-1} X(l) e^{j 2 \pi \frac{k l}{N}}$

For $\mathrm{N}=4$

$x(k)=\frac{1}{4} \sum_{l=0}^{3} X(l) e^{j \frac{\pi}{2} k l}$ for $\mathrm{k}^{\text {th }}$ sample

$x(3), x(0), x(1), x(2), x(3)$

$x(3)$ is the prefix and known as cyclic prefix CP

As we know that

$y(k)=h(0) x(k)+h(1) x(k-1)+v(k)$

Therefore,

$y(0)=h(0) x(0)+h(1) x(3)+v(0)$

$y(1)=h(0) x(1)+h(1) x(0)+v(1)$

$y(2)=h(0) x(2)+h(1) x(1)+v(2)---------$

$y(3)=h(0) x(3)+h(1) x(2)+v(3)$

This is circular shift of the channel taps or channel filter over sample $x(0), x(1), x(2), x(3)$.

Therefore output will be $y=h \otimes x+v$. Addition of cyclic prefix has converted linear convolution to circular convolution. In frequency domain circular convolution becomes product in FFT domain [4].

$\mathrm{N}$ point FFT at receiver

$$
\begin{aligned}
& Y(l)=\sum_{k=0}^{N-1} y(k) e^{-j 2 \pi \frac{k l}{N}} \text { If we put } \mathrm{N}=4 \\
& Y(l)=\sum_{k=0}^{3} y(k) e^{-j \frac{\pi}{2} k l}--------
\end{aligned}
$$

Therefore, received symbol across subcarrier $\mathrm{L}=2,[h(0), h(1), 0,0]$. Which is known as padding with N-L zero's, $\mathrm{N}=4$ and $\mathrm{L}=2$. Channel coefficient across subcarrier L $[H(0), H(1), H(2), H(3)]$

$H(l)=\sum_{k=0}^{N-1} h(k) e^{-j 2 \pi \frac{k l}{N}}$ Put N=4 we will get 
Malik, P. K.

Tripathi, M. P.

$H(l)=\sum_{k=0}^{3} h(k) e^{-j \frac{\pi}{2} k l}$

$[v(0), v(1), v(2), v(3)]$ With $\mathrm{N}=4$ pt DFT will gives

$[V(0), V(1), V(2), V(3)]$ These are noise samples across subcarrier.

$V(l)=\sum_{k=0}^{N-1} v(k) e^{-j 2 \pi \frac{k l}{N}}$

Put $\mathrm{N}=4$ in equation 7

$V(l)=\sum_{k=0}^{3} v(k) e^{-j \frac{\pi}{2} k l}$

Along L subcarrier

$Y(l)=H(l) \times X(l)+V(l)$

$\left[\begin{array}{l}Y(0) \\ Y(1) \\ Y(2) \\ Y(3)\end{array}\right]=\left[\begin{array}{l}X(0), 0,0,0 \\ 0, X(1), 0,0 \\ 0,0, X(2), 0 \\ 0,0,0, X(3)\end{array}\right]\left[\begin{array}{l}H(0) \\ H(1) \\ H(2) \\ H(3)\end{array}\right]+\left[\begin{array}{c}V(0) \\ V(1) \\ V(2) \\ V(3)\end{array}\right]$

$Y_{h}(l)=$ output symbol across subcarrier $\mathrm{L}$

$H_{h}(l)=$ Channel coefficient across subcarrier $\mathrm{L}$

$X(l)=$ Symbol loaded onto subcarrier $\mathrm{L}$

$V_{h}(l)$ Noise across subcarrier L

Here number of subcarrier is $\mathrm{N}=4$. $\mathrm{L}=0,1,2,3$

$Y_{h}(l)=H_{h}(l) \times X(l)+V_{h}(l)$

For $\mathrm{L}=0,1,2$ and 3

$Y(0)=H(0) \times X(0)+V(0)$

$Y(1)=H(1) \times X(1)+V(1)$

$Y(2)=H(2) \times X(2)+V(2)$

$Y(3)=H(3) \times X(3)+V(3)$

From equation 10 is it clear that no ISI interface from previous symbol on each subcarrier, ISI has been removed in frequency domain.

$H_{h}(n)=\frac{Y_{h}(n)}{X_{h}(n)}-----------------------(11)$

$[H(0), H(1), H(2), H(3)]$ with $\mathrm{N}=4 \mathrm{pt}$ IFFT will gives $[h(0), h(1), 0,0]$ with $\mathrm{N}$ $=4 \mathrm{pt}$ DFT 


$$
h(k)=\frac{1}{4} \sum_{l=0}^{3} H(l) e^{j \frac{\pi}{2} k l}
$$

OFDM: A

Mathematical

Review

\section{IFT AND FFT IN OFDM}

Two main key aspects of OFDM are FFT (Fast Fourier transform) and IFT (Inverse fast Fourier transform). Net transmitted MCM signal will be sum of transmit signals across all N subcarrier will be $x(t)=\sum_{k} X_{k} e^{j 2 \pi k F_{0} t}$

$\mathrm{Xk}$ is the symbol transmitted on kth subcarrier. $e^{j 2 \pi k F_{0} t}$ is the kth subcarrier. $k F_{0}$ is the $\mathrm{k}^{\text {th }}$ subcarrier frequency. Total number of subcarriers will be N. As $x(t)=\sum_{k} X_{k} e^{j 2 \pi k F_{0} t}$ generating this signal will be difficult because of large number of subcarrier [5]. Because for each subcarrier we need an oscillator i.e. for 100 subcarrier we will be needed 100 oscillators and should be preciously place.

As we know that $e^{j 2 \pi(k-l) F_{0} t}=\left\{\begin{array}{l}1, k=l \\ 0, k \neq l\end{array}\right.$ this principle is known as orthoganility principle. Which means that any two subcarrier $\mathrm{k}$ and 1 in this system is orthogonal.

How to overcome the problem, of large number of subcarrier can be explain as follows. Let a signal be a maximum frequency of $\mathrm{B}$ hence $F_{\max }=B / 2$ and $F_{\max }=B / 2$. As this is a band limited signal I can sample it at Nyquist rate. Nyquist rate is $2 F_{\max }=B$. Sampling duration or sampling interval $=1 /($ Sampling frequency $)=1 / \mathrm{B}=\mathrm{T} . \quad l^{\text {th }}$ sampling instant $=l T=\frac{1}{B}$ Lets come back to $x(t)=\sum_{k} X_{k} e^{j 2 \pi k F_{0} t}$ in this $l^{t h}$

sample will be $x(t)=\sum_{k} X_{k} e^{j 2 \pi k F_{0} t}$

$$
\begin{aligned}
& x(l)=x(l T)=\sum_{k} X_{k} e^{j 2 \pi k F_{0} l T} \\
& x(l)=x(l T)=\sum_{k} X_{k} e^{j 2 \pi \frac{k l}{N}}-
\end{aligned}
$$

Equation 14 says that this is exactly the expression of the IFFT. Which is $l^{\text {th }}$ IDFT point of $X(0), X(1), \ldots \ldots . . X(N-1) \quad X(0), X(1), \ldots \ldots . . X(N-1)$ are different symbols transmitted on $\mathrm{N}^{\text {th }}$ subcarrier. This can be easily generated with the help of $\mathrm{N}$ bank modulators. $x(0), x(1) \ldots \ldots x(N-1)$ are the samples of the 
Malik, P. K.

Tripathi, M. P. transmitted signals. $X(0), X(1) \ldots \ldots . X(N-1)$ are the transmitted symbols on transmitted samples $\mathrm{x}(0)$ signals.

That says

$X(0), X(1) \ldots . . . X(N-1)$ Transmitted symbols

$x(0), x(1) \ldots \ldots x(N-1)$ Transmitted samples (Sampled version of the MCM channel, which are transmitted over the wireless channel).

Similarly

$y(0), y(1) \ldots . . y(N-1)$

$\mathrm{N}$ point $\mathrm{FFT}$ will gives

$Y(0), Y(1) \ldots \ldots . . .(N-1)$

We have to send symbols that are to be transmitted on $\mathrm{N}$ sub carrier, we are performing the IFFT operation and generating the samples of the transmitted signals. . At the receiver we have received samples on these received samples we perform the FFT operation and then reconstructed the originals symbols . This the key aspect of the OFDM [6].

\section{CYCLIC PREFIX IN OFDM}

Let us consider a frequency selective channel model of frequency $y(n)=h(0) x(n)+h(1) x(n-1)+\ldots \ldots . . h(L-1) x(n-L+1)$

Which has channel taps $h(0), h(1), h(2) \ldots \ldots . . . h(L-1)$, which says that $\mathrm{L}$ channel taps. From the above equation it can be shown that output does not depend on current input but also on previous input $x(n-1)$. Therefore in the time domain this channel has inter symbol interference (ISI ). Therefore, this input output received symbol model consists of ISI.

Now let us consider the output of the OFDM system. Output for $\mathrm{x}(0)$ will be $y(0)=h(0) x(0)+h(1)$

But if we consider previous symbol. Output for $\mathrm{x}(0)$ will be $y(0)=h(0) x_{h}(0)+h(1) x_{h}(N-1)+\ldots . . h(L-1) x_{h}(N-L+1)$

Now there is a problem, we have inter-block interference. We have to remove these inter block interference in OFDM. To remove inter block interference we use cyclic prefix. As we know that $X(0), X(1) \ldots \ldots . . X(N-1)$ Transmitted symbols 
$\mathrm{N}$ point IFFT

$$
x(N-\bar{L}), \ldots \ldots x(N-2), x(N-1), x(0), x(1) \ldots \ldots x(N-1)
$$

Transmitted samples of OFDM system (Sampled version of the MCM channel, which is transmitted over the wireless channel). Prefix of L bar samples from the tail.

Now instead of directly transmitting these samples, we are going to add a prefix. We are taking L bar samples.

$X(0), X(1) \ldots . . X(N-1)$ Transmitted symbols

$\mathrm{N}$ point IFFT

$$
x(N-\bar{L}), \ldots \ldots x(N-2), x(N-1), x(0), x(1) \ldots \ldots x(N-1)
$$

Now if you look at the first symbol: -

$$
y(1)=h(0) x(1)+h(1) x(0)+\ldots . . h(L-1) x(N-L+1)
$$

Now the interference has been restricted to the same block. Therefore we have avoided inter block interference. There is ISI but there is no inter block interference. Similarly $y(1)=h(0) x(1)+h(1) x(0)+\ldots . . h(L-1) x(N-L+1)$

This gives us a very important concept, addition of cyclic prefix (CP), has resulted in a circular convolution at the output of the OFDM system. Therefore, $y=h \otimes x$

$\mathrm{y}$ is the received OFDM samples, $\mathrm{h}$ is the channel coefficient and $\mathrm{x}$ is the transmitted OFDM samples. We know that if $y=h \otimes x$, then: $Y(k)=H(k) X(k)$ Because circular convolution in the time domain is the multiplication in the frequency domain.

$\mathrm{X}(\mathrm{k})$ is the $\mathrm{K}^{\text {th }}$ DFT coefficient of the transmitted signal

$\mathrm{Y}(\mathrm{k})$ is the $\mathrm{K}^{\text {th }} \mathrm{DFT}$ coefficient of the received signal

$\mathrm{H}(\mathrm{k})$ is the $\mathrm{K}^{\text {th }}$ DFT coefficient of the transmitted signal

\section{BER PERFORMANCE OF OFDM}

Generalize system model can be expressed as received sample $y=h \otimes x+w$. $\mathrm{w}$ is Gaussian noise. Once we take the FFT at the receiver we have $Y(k)=H(k) X(k)+W(k)$

$\mathrm{Y}(\mathrm{k})$ is the kth FFT point of the received samples $[\mathrm{y} 0, \mathrm{y} 1, \ldots . . \mathrm{y}(\mathrm{n}-1)]$

$\mathrm{W}(\mathrm{k})$ is the kth FFT point of the noise samples $[\mathrm{w} 0, \mathrm{w} 1, \ldots . . \mathrm{w}(\mathrm{n}-1)]$

In time domain we have the received signal $y=h \otimes x+w$
OFDM: A

Mathematical

Review 
Malik, P. K.

Tripathi, M. P.

In other words $W(k)=\sum_{l=0}^{N-1} w(l) e^{-j 2 \pi \frac{k l}{N}}$

Each w(1) is IID Gaussian in nature with zero mean, therefore.

Mean

$E\{w(l)\}=0$

Variance

$E\left\{|w(l)|^{2}\right\}=\sigma^{2}$

Covariance

$E\left\{w(l) w^{*}(\tilde{l})\right\}=0$

Now let us look the property of $W(k)=\sum_{l=0}^{N-1} w(l) e^{-j 2 \pi \frac{k l}{N}}$

$E\{W(k)\}=\sum_{l=0}^{N-1} E\{w(l)\} e^{-j 2 \pi \frac{k l}{N}}$

$E\{W(k)\}=0$

Which says that expected value of each $\mathrm{W}(\mathrm{k})$ is zero. Since the FFT is a linear combination, the input of noise samples is zero mean. Naturally at the output of the FFT W(k) is also zero mean.

Let us look the variance of each noise samples.

$E\left\{|W(k)|^{2}\right\}=$ ?

$E\left\{W(k) \cdot W^{*}(k)\right\}=E\left\{\left(\sum_{l=0}^{N-1} w(l) e^{-j 2 \pi \frac{k l}{N}}\right)\left(\sum_{l=0}^{N-1} w(\tilde{l}) e^{-j 2 \pi \frac{k l}{N}}\right)^{*}\right\}$

$E\left\{W(k) \cdot W^{*}(k)\right\}=E\left\{\sum_{l=0}^{N-1} \sum_{i=0}^{N-1} w(l) w^{*}(\tilde{l}) e^{-j 2 \pi \frac{k(l-\tilde{l})}{N}}\right\}---(19)$

Noise sample w(l) are independent and therefore uncorrelated. Therefore expected value will survive only if $l=\tilde{l}$.

$$
\begin{aligned}
& E\left\{W(k) \cdot W^{*}(k)\right\}=\left\{\sum_{l=0}^{N-1} \sum_{i=0}^{N-1} E\left\{w(l) w^{*}(\tilde{l})\right\}\right\} e^{-j 2 \pi \frac{k(l-\tilde{l})}{N}} \\
& E\left\{w(l) w^{*}(\tilde{l})\right\}=\left\{\begin{array}{l}
0 \ldots i f, l \neq \tilde{l} \\
\sigma^{2} \ldots i f f, l=\tilde{l}
\end{array}\right\}------------
\end{aligned}
$$




$$
\begin{aligned}
& E\left\{W(k) \cdot W^{*}(k)\right\}=\sum_{l=0}^{N-1} \sigma^{2} \cdot 1 \\
& E\left\{W(k) \cdot W^{*}(k)\right\}=N \sigma^{2}---
\end{aligned}
$$

Hence

$$
E\left\{|W(k)|^{2}\right\}=N \sigma^{2}
$$

Therefore, noise at the output can be characterize as

Mean $E\{W(k)\}=0$ Variance $E\left\{|W(k)|^{2}\right\}=N \sigma^{2}$

Similarly for channel coefficient: Each $\mathrm{h}(1)$ is Rayleigh fading coefficient $h(0), h(1), \ldots \ldots . h(L-1), 0,0,0$

$H(k)=\sum_{l=0}^{L-1} h(l) e^{-j 2 \pi \frac{k l}{N}}$

Each $\mathrm{h}(1)$ is Rayleigh fading coefficient, which mean each $h(1)$ is complex Gaussian with mean zero. $E\{h(l)\}=0 \quad E\left\{h(l) \cdot h^{*}(\tilde{l})\right\}=0$ if $l \neq \tilde{l}$

Each $\mathrm{h}(1)$ are uncorrelated. This is known as uncorrelated scattering assumption. Similarly power of each Rayleigh fading is $E\left\{|h(l)|^{2}\right\}=1$

If $h(l)$ is Rayleigh in nature then $H(k)$ will also be Rayleigh in nature. i.e.

$$
\begin{aligned}
& E\{H(k)\}=0 \\
& E\left\{|H(k)|^{2}\right\}=\sum_{l=0}^{L-1} E\left\{|h(l)|^{2}\right\} \\
& E\left\{|H(k)|^{2}\right\}=\sum_{l=0}^{L-1} 1 \\
& E\left\{|H(k)|^{2}\right\}=L-----
\end{aligned}
$$

Now if we see our system model across subcarrier

$$
Y(k)=H(k) X(k)+N(k)
$$

$H(k)$ is Rayleigh fading with average power (Variance) L $E\left\{|H(k)|^{2}\right\}=L$ $N(k)$ with average power (variance) $N \sigma^{2} E\left\{|W(k)|^{2}\right\}=N \sigma^{2}$

Let us assume $X(k)$ with average power (Variance) be $\mathrm{P} E\left\{|X(k)|^{2}\right\}=P$ Therefore SNR for this problem will be $S N R=\frac{|H(k)|^{2} P}{N \sigma^{2}}$ at the receiver and average of the received power at the receiver $S N R_{r}=\frac{|H(k)|^{2} P}{N \sigma^{2}}=\frac{L P}{N \sigma}$. Similarly as the chan- 
Malik, P. K.

Tripathi, M. P. nel Rayleigh fading BER of OFDM for BPSK transmission will be

$$
B E R=\frac{1}{2}\left(1-\sqrt{\frac{\frac{L}{N} S N R}{2+\frac{L}{N} S N R}}\right)
$$

\section{RESULT}

A MATLAB based program is written to compute the above specified methods and following are the results of the program. For simplicity of the data presentation, following terms are assumed. Number of bits per channel is 32 but can be extended upto 128 Number of subcarrier channel is 4 Total number of bits to be transmitted at the transmitter is 128 Size of each OFDM block to add cyclic prefix 16 Channel is AWGN in nature Random samples are taken for the transmission purpose. These samples are in the binary form. Sample size is kept to be 32 .

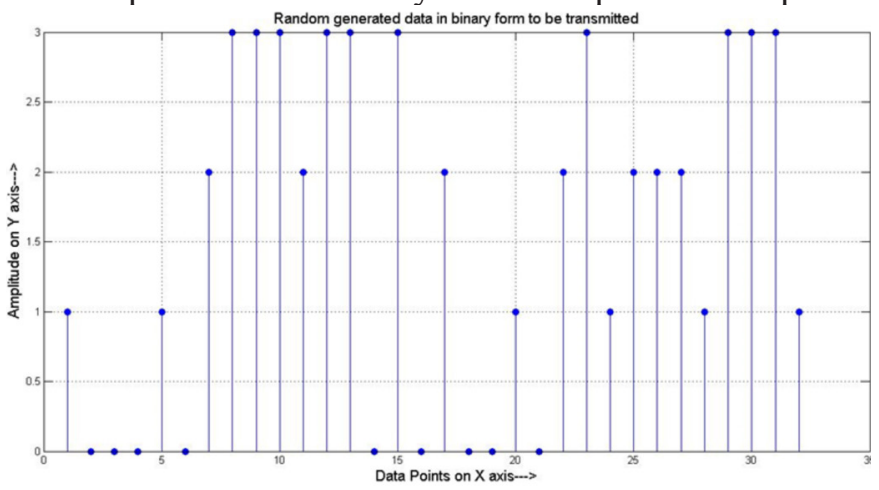

Fig 1: Random generated data to be transmitted

Quadrature phase shift keying method is employed here to modulate the binary signal. Following graph shows the output of the QPSK modulator.

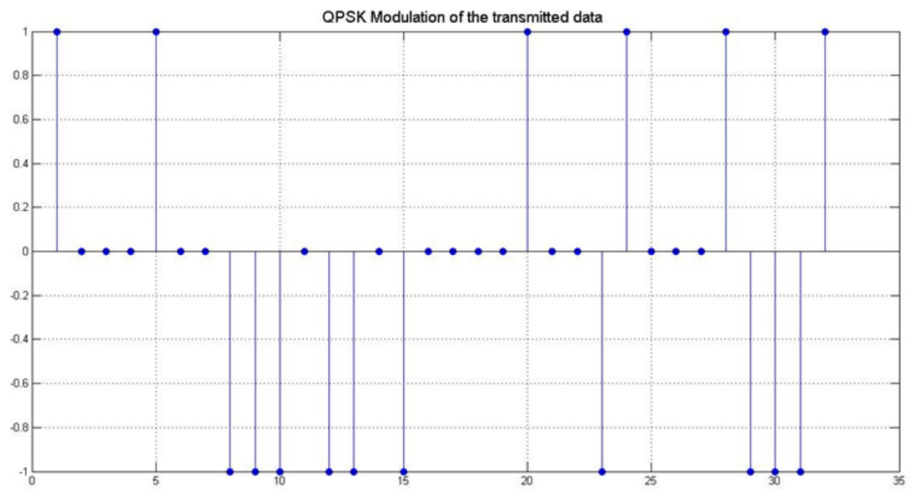

Fig 2: QPSK Modulation of the data to be transmitted 
Before the transmission of the data IIFT is done for all sub carriers.
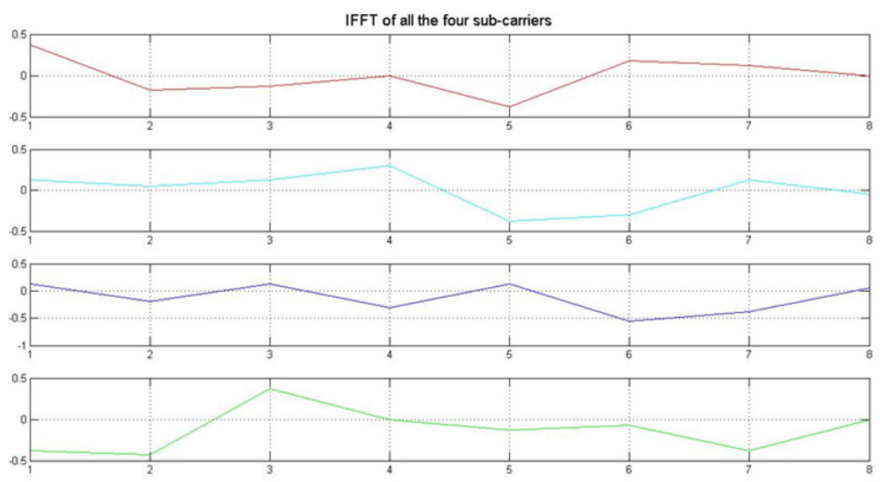

Fig 3: IFFT of sub carrier at transmitter

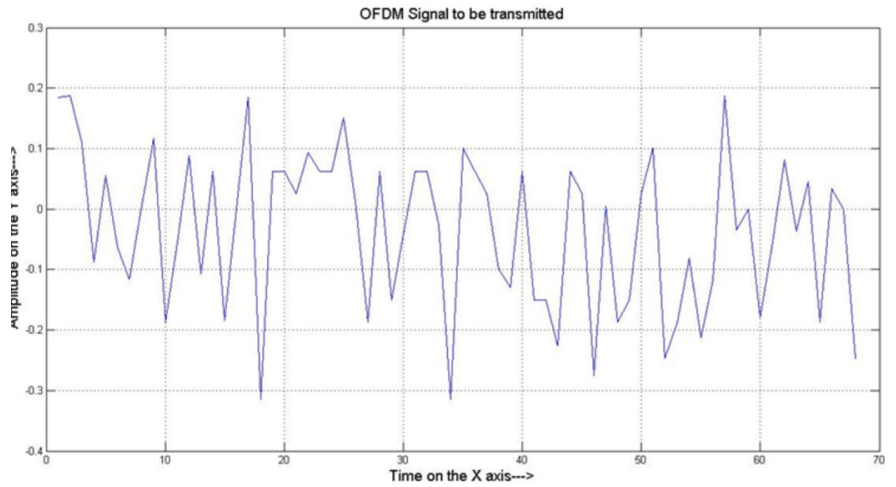

Fig 4: Transmitted OFDM signal

Following graph shows the output of the receiver at the receiver. i.e received OFDM signal.

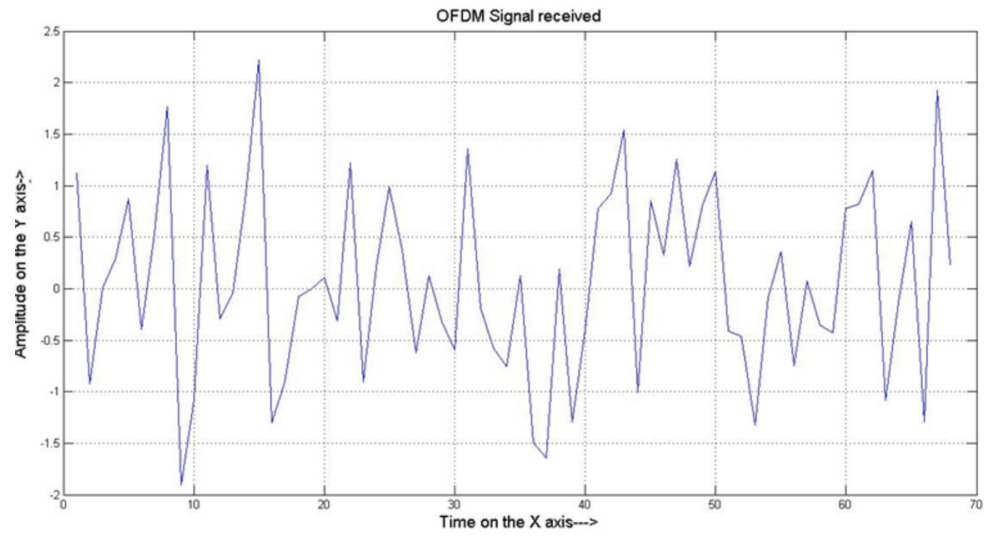

Fig 5: Received OFDM signal 
Malik, P. K.

Tripathi, M. P.
Fast Fourier transform is employed on the received signal for all sub carriers.
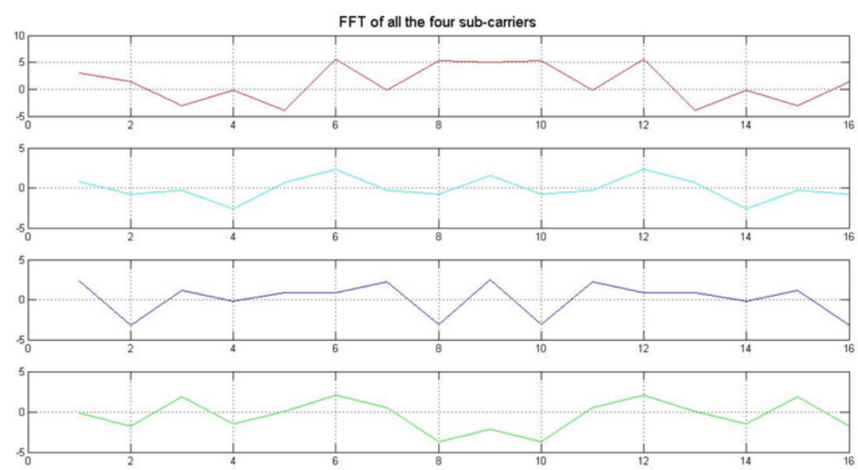

Fig 6: FFT of received signal

As the output of the FFT we received the original signal, in this case it seems that it is almost similar to the input.

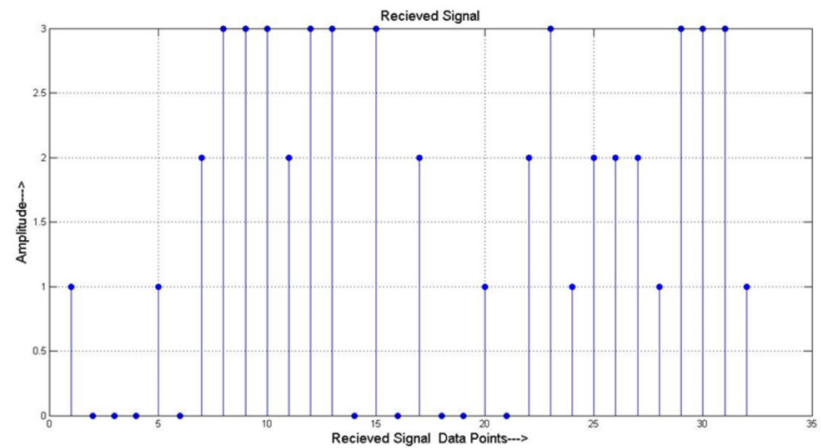

Fig 7: Received signal at the receiver in binary form

For the proposed statistically computed BER Vs SNR of the OFDM system, it has been observed that it is continuously decreasing as a function of SNR.

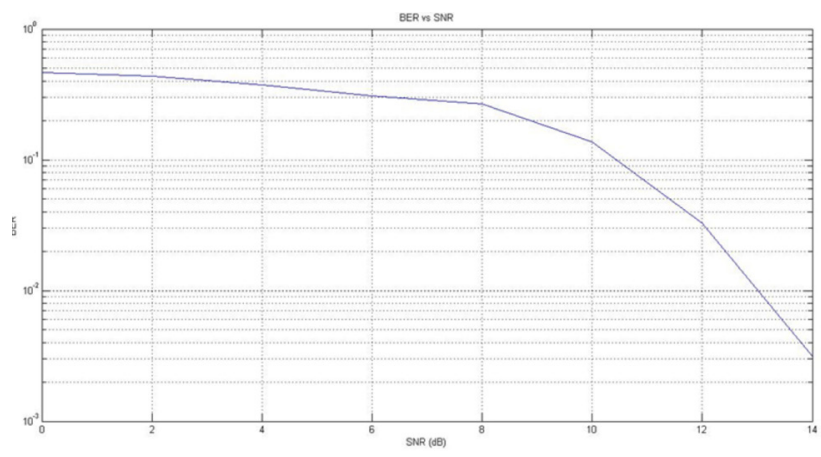

Fig 8: BER Vs SNR plot 


\section{ACKNOWLEDGMENT}

Author would like to thanks Prof. Harish Parthsarthy, Dept. of Electronics and Communication, N.S.I.T, New Delhi for his support. I feel grateful for the support of Prof. M P Tripathi, Emirates Prof. NIT Patna, for writing and publishing paper. The author will also like to express sincere appreciation and gratitude to department of Electronics and Communication, Inderprastha Engineering College, Ghaziabad UP India, which has provided tremendous assistance throughout the work

\section{REFERENCES}

1. J.A. C. Bingham (1990) "Multicarrier modulation for data transmission: An idea whose time has come,” IEEE Commun. Mag., vol. 28, pp. 5-14. http://dx.doi.org/10.1109/35.54342

2. R. van Nee and R. Prasad (2000) "OFDM for Wireless Multimedia Communications" Boston: Artech House.

3. R. W. Chang (1966) "Synthesis of band limited orthogonal signals for multichannel data transmission,” Bell Labs Tech. Journal, Vol. 45, Issue 10, pp. 1775-1796. http://dx.doi. org/10.1002/j.1538-7305.1966.tb02435.x

4. W. Shieh and I. Djordjevic (2010) "Orthogonal Frequency Divi- sion Multiplexing for Optical Communication," Acade- mic Press, Waltham.

5. B. Saltzberg (1967) "Performance of an efficient parallel data transmission system," IEEE Transactions on Communications, IEEE, Vol. 15, Issue 6, pp. 805-811.

6. J. Ketonen, M. Juntti and J. R. Cavallaro (2010) "Performance Complexity Comparison of Receivers for a LTE MIMO-OFDM System ”, IEEE Transaction on Signal Processing, Vol. 58, No. 6, pp. 3360 - 3372. http://dx.doi.org/10.1109/TSP.2010.2044290.
OFDM: A

Mathematical

Review 